\title{
Physics potential of the Jiangmen Underground Neutrino Observatory
}

\author{
C. Lombardo, R. Caruso, S. Costa, A. Insolia, C. Tuvé, N. Giudice, N. Guardone \\ INFN section of Catania, Department of Physics and Astronomy "Ettore Majorana", University \\ of Catania, Italy \\ E-mail: claudio.lombardo94@outlook.it
}

M.Buscemi, G.Andronico*, S.Aiello, R.Bruno, M.Fargetta, S.Monforte, G.Verde INFN section of Catania

E-mail: giuseppe.andronico@ct.infn.it

\section{On behalf of the JUNO Collaboration}

\begin{abstract}
JUNO (Jiangmen Underground Neutrino Observatory) is a 20 kton multipurpose neutrino detector under construction in China and it will be completed in 2021. The detector will be located near the the city of Jiangmen, $53 \mathrm{~km}$ away from two nuclear power plants that will provide the neutrino flux for the main goal of the observatory.

The main physics goal is the determination of the neutrino Mass Hierarchy, even if will play an important role also in other topics, such as: neutrino oscillation, solar neutrino, SuperNova neutrinos, geoneutrinos and atmosperic neutrinos among others.
\end{abstract}

XXIX International Symposium on Lepton Photon Interactions at High Energies - LeptonPhoton2019 August 5-10, 2019

Toronto, Canada

${ }^{*}$ Speaker. 


\section{Introduction}

The determination of the correct mass hierarchy plays an important role on neutrino physics. For example it is important in Dirac or Majorana mass terms, specially after the high precision measurements performed on the oscillation parameters.

The fact that neutrinos can oscillate from a lepton family to another during its propagation is a direct consequence of a non-zero neutrino mass, as well as the existence of a mass hierarchy itself. There are three neutrino mass eigenstates: $v_{1}, v_{2}, v_{3}$, different from the three $v_{e}, v_{\mu}$, and $v_{\tau}$ lepton eigenstates. However, it's not known if the eigenstate $v_{3}$ is heavier or lighter than $v_{1}$ and $v_{2}$. In fact, it's known that $m_{2}>m_{1}$ and $\left|\Delta m_{31}^{2}\right| \gg\left|\Delta m_{21}^{2}\right|$ where $\Delta m_{i j}^{2}=m_{i}^{2}-m_{j}^{2}$. What is not known is the absolute scale of the mass hierarchy namely whether $m_{3}$ is larger than $m_{1}$ and $m_{2}$ ("Normal" Hierarchy) or $m_{3}$ is smaller than $m_{1}$ and $m_{2}$ ("Inverted" hierarchy).

Furthermore, there are other neutrino aspects that require further study: high precision measurements of the oscillation parameters, measurement of geoneutrino flux, measurement of the neutrinos generated during a core collapse SuperNova and measurement on the Diffusive Neutrino SuperNova background (neutrino generated in old SuperNova's explosions)

\section{JUNO detector}

The JUNO detector [1] will be located around $700 \mathrm{~m}$ under sea level and $53 \mathrm{~km}$ away from two nuclear power plants characterized by a total power of about $36.6 \mathrm{GW}$, which will provide a high flux of electron antineutrino.

The detector is made up by an acrylic ball filled up with a liquid scintillator, linear alkylbenzene (LAB). The sphere is immersed in a tank filled up with pure water. This tank is very important for two reasons: first, it protects the main detector from fast neutron produced during natural radioactive decay in the environment and, second, it will be read-out by 2000 20" PMTs to be used as veto detector for muons which produce Cherenkov light during their passage in water. A plastic scintillator detector will be on the top outer part of the detector, to be used as another veto detector for muons produced in the atmosphere. There are two main channels to identify neutrino events: Inverse Beta Decay $\left(\bar{v}_{e}+p \rightarrow e^{+}+n\right)$ and elastic scattering $\left(\bar{v}_{e}+e^{-} \rightarrow \bar{v}_{e}+e^{-}\right)$. The key to determine the correct hierarchy is the energy resolution, for which the JUNO goal is $\frac{3 \%}{\sqrt{E[\mathrm{MeV}]}}$.

\section{JUNO physics}

\subsection{Mass Hierarchy}

Atmospheric mass squared difference manifest itself cyclically in the energy spectrum. Therefore, comparing the experimental spectrum with the theoretical one, JUNO will extract information about the hierarchy by means of a $\chi^{2}$ test. The effect of the neutrino mass hierarchy on the electron antineutrino energy spectrum is shown in Fig. 1, where the Normal mass order corresponds to the blue line and the Inverted one corresponds to the red line.

The high energy resolution demand on JUNO is expected to resolve the energy spectrum to the point of answering the question about absolute mass ordering. 


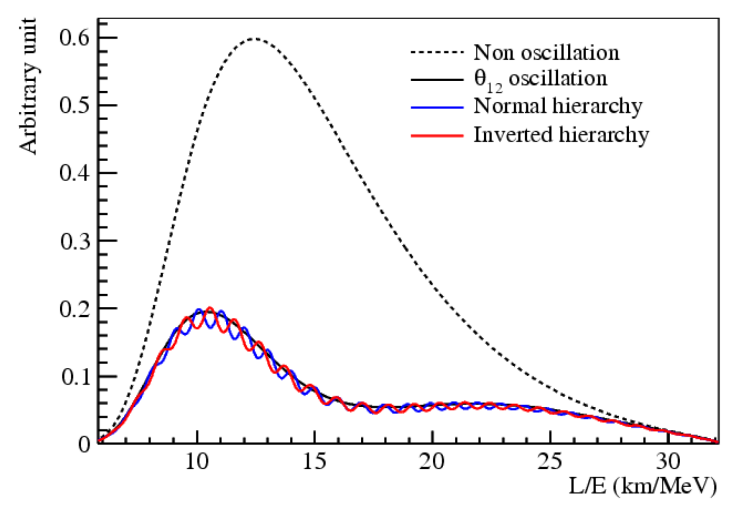

Figure 1: The un-oscillated spectra of reactor anti-neutrino (in black) at the distance of $53 \mathrm{~km}$ and the oscillated spectra for the two mass orderings (blue is the normal order and red is the inverted order)[1].

\subsection{SuperNova Neutrino}

Another important aim for JUNO consists of the detection of neutrinos coming from astrophysical sources, in particular SuperNova neutrino burst and diffusive flux. JUNO can detect SN v's via different channels: inverse beta decay, is the "golden" channel with $\sim 5000$ expected events[2], while $v$-p elastic scattering can account for about $\sim 1000$ events [3] and $v$-e elastic scattering can provide about $\sim 300$ events [3]. The number of events is indicated for a core collapse SuperNova at $10 \mathrm{kpc}$ away the Earth. In Fig. 2 is shown the all channels event rates for two different models for a core collapse SuperNova, 1D model on the left panels and the 2D model on the right panels. The event rates are calculated for a progenitor of 10 solar masses, top panels, and 19 solar masses, bottom panels. Rates are also shown in the case where there was no oscillation, red line, in the case where the mass hierarchy was Normal, blue line, or if it was Reverse, green line.

\subsection{Geoneutrinos}

Measuring the $\bar{v}_{e}$ emitted in the ${ }^{238} U$ and ${ }^{232}$ Th radioactive decay chains (called geoneutrinos), helps in testing the abundance of Thorium and Uranium present in the Eart's nucleus and in the Earth's mantle, in particular JUNO will estimate Th/U rate. This is important to understand the contribution to the radiogenic heat. In Fig. 3 data about signal from different Earth components and ratio between different neutrino sources.

\subsection{Dark Matter searches}

Resonant interactions between neutrinos from a Galactic supernova and dark matter particles can lead to a sharp dip in the neutrino energy spectrum. Due to its excellent energy resolution, measurement of this effect can provide evidence for such couplings.

\subsection{Solar Neutrinos}

The JUNO detector has many advantages in performing solar neutrinos measurements. It has the benefit of high light yield, very high energy resolution and low energy threshold. Thanks to this 

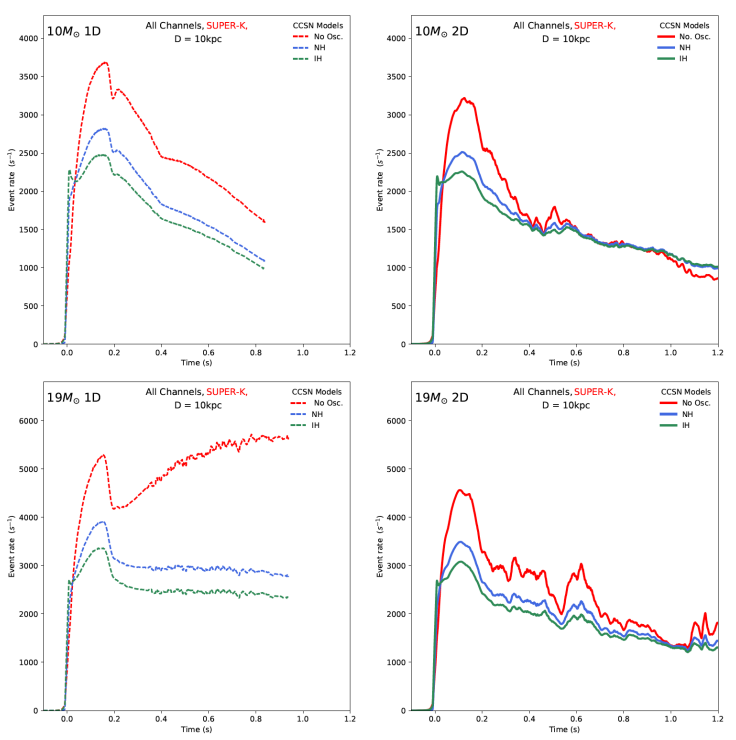

Figure 2: All channels event rates (in units of $s^{-1}$ ) for a SN at $10 \mathrm{kpc}$ for the $1 \mathrm{D}$ model for all 3 flavours oscillations theory (left) and 2D model (right). This is shown for a progenitor of 10 solar masses (top plots) and for a progenitor of 19 solar masses (bottom plots)[4].
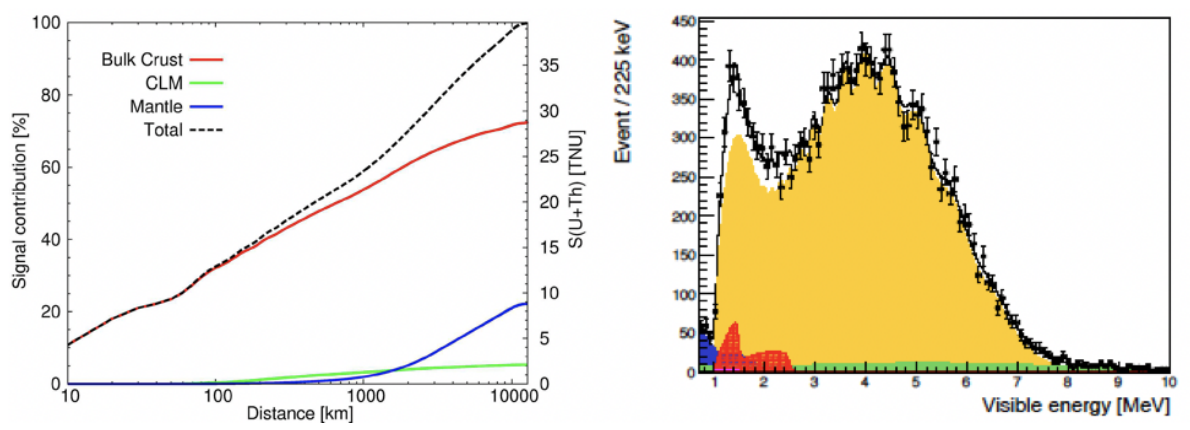

Figure 3: On left panel the contribution in the signal from different Earth components in function of distance from JUNO. On right panel IBD signal (simulated) after 1 year: in red geoneutrinos signal, in yellow signal from reactor neutrinos while in green background from $\mathrm{Li}$ and in blue background from accidental events [2].

features JUNO will remeasure the flux of neutrinos produced by the decay chain of ${ }^{7} \mathrm{Be}$ and ${ }^{8} \mathrm{~B}$. In Fig. 4 expected JUNO spectra relevant to solar neutrinos.

\section{Conclusion}

The JUNO detector, due to its high energy resolution, has a high potential to determine the neutrino mass hierarchy using the oscillation of electron antineutrinos generated by fission processes occuring in two nuclear power plants. Besides its main physics goal, it will also be able to precisely measure the neutrino oscillation parameters and address important questions in astrophysical neutrino and geoneutrinos. 


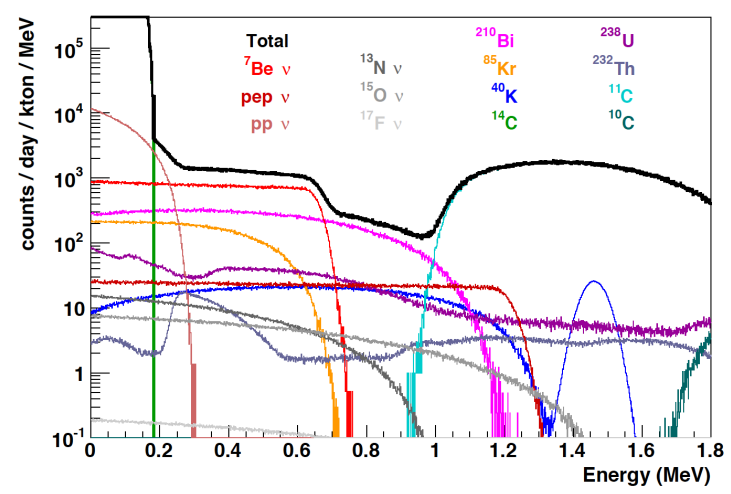

Figure 4: Expected singles spectra at JUNO [2].

\section{References}

[1] Giaz, Agnese. "Status and perspectives of the JUNO experiment." arXiv:1804.03575 (2018).

[2] An, Fengpeng, et al. "Neutrino physics with JUNO.", Journal of Physics G: Nuclear and Particle Physics 43.3 (2016): 03040.

[3] Miramonti, L. Neutrino Physics and Astrophysics with the JUNO Detector, Universe 4.11 (2018): 126.

[4] Seadrow, S. et al. Neutrino signals of core-collapse supernovae in underground detectors.Monthly Notices of the Royal Astronomical Society 480.4 (2018): 4710-4731 